\title{
Variations of OCT measurements corrected for the magnification effect according to axial length and refractive error in children
}

\author{
Inmaculada Bueno-Gimeno*, ${ }^{*}$, Enrique España-Gregori ${ }^{\dagger}$, Andres Gene-Sampedro*, \\ Juan Carlos Ondategui-Parra ${ }^{\star}$ and Carlos J. Zapata-Rodriguez* \\ *Department of Optics and Optometry and Vision Science \\ University of Valencia, Spain \\ ${ }^{\dagger}$ Department of Surgery, University of Valencia \\ University Hospital La Fe, Spain \\ $\star$ Faculty of Optics and Optometry, University Vision Centre \\ Polytechnic University of Catalonia, Spain \\ \$inmaculada.bueno@uv.es
}

Received 27 March 2017

Accepted 30 June 2017

Published 26 July 2017

\begin{abstract}
Purpose: The aim of this paper was to examine the distribution of macular, retinal nerve fiber layer (RNFL) thickness and optic disc parameters of myopic and hyperopic eyes in comparison with emmetropic control eyes and to investigate their variation according to axial length (AL) and spherical equivalent (SE) in healthy children. Methods: This study included 293 pairs of eyes of 293 children (145 boys and 148 girls), ranging in age from 6 to 17 years. Subjects were divided according to SE in control (emmetropia, 99 children), myopia (100 children) and hyperopia (94 children) groups and according to axial AL in 68 short $(<22.00 \mathrm{~mm}, 68)$, medium (from $\geq 22.00 \mathrm{~mm}$ to $25.00 \mathrm{~mm}, 189)$ and long eyes $(>25.00 \mathrm{~mm}, 36)$. Macular parameters, RNFL thickness and optic disc morphology were assessed by the Cirrus ${ }^{\mathrm{TM}}$ HD-OCT. AL was measured using the IOL-Master system. Littmann's formula was used for calculating the corrected AL-related ocular magnification. Results: Mean age $( \pm \mathrm{SD})$ was $10.84 \pm 3.05$ years; mean $( \pm \mathrm{SD}) \mathrm{SE}$ was $+0.14 \pm 0.51 \mathrm{D}$ (range from -8.75 to $+8.25 \mathrm{D})$ and mean $\mathrm{AL}( \pm \mathrm{SD})$ was $23.12 \pm 1.49$. Average RNFL thickness, average macular thickness and macular volume decreased as AL and myopia increased. No correlations between $\mathrm{AL} / \mathrm{SE}$ and optic disc parameters were found after correcting for magnification effect. Conclusions: AL and refractive error affect measurements of macular and RNFL thickness in healthy children. To make a correct interpretation of OCT measurements, ocular magnification effect should be taken into account by clinicians or OCT manufacturers.
\end{abstract}

Keywords: Axial length; refractive error; children; OCT measurements; magnification effect.

$\S_{\text {Corresponding author. }}$

This is an Open Access article published by World Scientific Publishing Company. It is distributed under the terms of the Creative Commons Attribution 4.0 (CC-BY) License. Further distribution of this work is permitted, provided the original work is properly cited. 


\section{Introduction}

Optical coherence tomography (OCT) is a noninvasive, noncontact technology that provides crosssectional images of the retina. The instrument uses specific algorithms to automatically measure retinal thickness (RT) and retinal nerve fiber layer (RNFL), to quantify optic nerve head (ONH) morphology and to assess changes in retinal diseases. ${ }^{1}$ This is especially important in children because visual loss associated with retinal disease can negatively influence their visual development. ${ }^{2}$

Wang et $a l .{ }^{3}$ revealed that magnification attributable to axial length (AL) and refractive error has minimal impact on measurements of macular and RNFL thickness in 120 children, but they asserted that transverse measurements such as optic disc diameter should be corrected by magnification effect. However, other studies conducted in adults ${ }^{4,5}$ and children ${ }^{6,7}$ found that $\mathrm{AL}^{4-7}$ and refractive error $^{5,6,8}$ influence the measurements of OCT. The studies mentioned earlier ${ }^{4-8}$ referred that AL-related ocular magnification should be taken into account to ensure better accuracy in the measurements made with OCT. Previous studies used time domain OCT (Stratus OCT); however, spectral domain (SD-OCT) such as Cirrus HD-OCT offers a higher axial resolution and scanning speed than conventional time-domain techniques. ${ }^{9}$ These OCT devices (Stratus and Cirrus OCT) did not apply the correction for magnification effect. As referred Ctori et al. ${ }^{10}$ ocular magnification of retinal images is affected by refractive error, corneal curvature, refractive index, AL and anterior chamber depth as well as the distance from the eye. Spectralis SDOCT employs an automatic modification process in order to neutralize the effect of ocular magnification. ${ }^{10}$ Ocular magnification is a factor of disagreement between both devices (Spectralis SD-OCT and Cirrus OCT) because of the scan path used. Spectralis SD-OCT uses a circular scan path, and Cirrus OCT interpolates the scan from a $200 \times 200$ A-scan cube centered on the optic nerve. ${ }^{6}$

Lim and $\mathrm{Chun}^{8}$ compared, in a sample of 35 children younger than 10 years, the peripapillary RNFL thickness, macular thickness and total macular thickness of high myopic eyes with a mean spherical equivalent $(\mathrm{SE})$ of $-7.93 \pm 1.46 \mathrm{D}$ with those of low myopic eyes with a mean SE of $-1.41 \pm 1.32 \mathrm{D}$, concluding that high myopic children had significantly lower values of overall peripapillary RNFL thickness, macular thickness and macular volumes than low myopic children. In this study, ${ }^{8}$ the authors corrected for ocular magnification effect. Taş et al. ${ }^{6}$ divided a sample of 164 hyperopic children into three groups according to their SE as low, moderate and high hyperopia group and found that the mean RNFL and the RNFL of inferior and nasal quadrants were thicker in children with high hyperopia than in children with low hyperopia. However, these differences disappeared after correction of magnification. ${ }^{6}$ Savini et al. ${ }^{4}$ used the magnification correction for evaluating RNFL thickness and $\mathrm{ONH}$ parameters (optic-disc area and rim area) in 45 healthy adults (mean age: $39.4 \pm 7.2$ years), dividing their sample into three groups according to AL as short, medium and long eyes. The authors revealed that AL influences measurements of both RNFL thickness and $\mathrm{ONH}$ parameters and suggested caution when measurements of myopic and hyperopic eyes were compared with the normative database of the instrument. ${ }^{4}$

Huynh et al. ${ }^{2}$ corrected in 1309 6-year-old children transverse disc OCT measurements for magnification and concluded that AL appears to have a stronger effect on disc and rim area than the refraction. All of the aforementioned studies utilized Stratus-OCT to take the measurements. As previously reported by Savini et al. ${ }^{4}$ the Cirrus HD-OCT and Stratus-OCT should behave similarly, when AL-induced ocular magnification is accounted for.

The aim of this study was to evaluate the distribution of macular and RNFL thickness and $\mathrm{ONH}$ parameters of myopic and hyperopic eyes in comparison with emmetropic control eyes and to investigate the variations of these parameters according to $\mathrm{AL}$ and refractive error in healthy Caucasian children. Measurements were performed with SD-OCT and corrected for magnification. To our knowledge, there are a small number of studies, which analyze the impact of both AL and refraction on OCT measurements in Caucasian children.

\section{Materials and Methods}

\subsection{Patients}

This was a cross-sectional study that included 293 eyes of 293 healthy children (145 boys and 148 girls) ranging in age from 6 to 17 years selected from a previous study database. ${ }^{11}$ The sample was classified according to the cycloplegic SE refraction of 
both eyes, and subjects were divided into three groups: 99 emmetropic eyes as a control group (45 boys and 54 girls), 100 myopic eyes ( 46 boys and 54 girls) and 94 hyperopic eyes (54 boys and 40 girls). Emmetropia was defined as a cycloplegic SE between +0.75 and $-0.25 \mathrm{D}$. $^{12}$ The myopic group was further categorized into three subgroups according to the SE: low myopia (SE between -0.50 and $-3.00 \mathrm{D}$ ), moderate myopia (SE between -3.25 and $-6.00 \mathrm{D}$ ) and high myopia (SE greater than -6.00 D). ${ }^{12,13}$ The hyperopic group was similarly categorized into low ( $\mathrm{SE}$ between +1.00 and $+3.00 \mathrm{D}$ ), moderate ( $\mathrm{SE}$ between +3.25 and $+6.00 \mathrm{D}$ ) and high (SE greater than +6.00 D) hyperopia. As the effect of AL on macular and RNFL thickness and optic disc parameters was investigated, our sample was also divided into three groups according to the magnitude of AL: short $(<22.00 \mathrm{~mm}, 68$ eyes, 33 boys and 35 girls), medium (from $\geq 22.00 \mathrm{~mm}$ to $25.00 \mathrm{~mm}, 189$ eyes, 88 boys and 101 girls) and long eyes groups ( $>25.00 \mathrm{~mm}, 36$ eyes, 19 boys and 17 girls). ${ }^{14}$

Exclusion criteria for the study were a corrected distance visual acuity worse than $20 / 25$ in either eye and a refractive cylinder of more than $2.00 \mathrm{D}$ because the degree of corneal astigmatism influences the $\mathrm{ONH}$ parameters and peripapillary RNFL thickness measurements by the Cirrus HDOCT. ${ }^{15,16}$ Subjects with a previous history of ocular surgery, trauma, pathology or ocular medication and those with tilted optic disc, anisometropia more than $1.00 \mathrm{D}$ or strabismus were also excluded from the study.

\subsection{Examination protocol}

All subjects underwent a comprehensive ocular examination that included visual acuity measurement, stereopsis assessment, motility exam, cycloplegic refraction, anterior segment and dilated fundus examination. Cycloplegia was induced with three drops of cyclopentolate $1 \%$ separated by $5 \mathrm{~min}$, to achieve adequate mydriasis $(\geq 6 \mathrm{~mm}){ }^{2}$ At least $30 \mathrm{~min}$ after the last drop, autorefraction was performed with an autorefractometer (Topcon KR-8100P), followed by a subjective refractive refinement.

The same experienced examiner (I. B.-G.) performed all of the measurements in both eyes in a random order 15 days after the initial ocular examination in order to avoid any effect induced by cycloplegia. Only data from one eye randomly selected in each patient were included in the study. The AL was measured using the IOL-Master system (Version 5.2.1, Carl Zeiss Meditec, Jena, Germany). ${ }^{17}$ Three consecutive AL readings were taken and averaged. Only the AL measurements with a signal-to-noise ratio greater than 2 were included in the database. ${ }^{13}$ All measurements were performed without pupil dilation. Intraocular pressure was determined and analyzed in a previous study, ${ }^{12}$ and all children included in the analysis were selfreported healthy.

The study followed the tenets of the Declaration of Helsinki, and informed written consent was obtained from the children's legal guardians.

\subsection{OCT (Cirrus $\left.{ }^{T M} H D-O C T\right)$ measurements}

Measurements of the RNFL thickness, ONH parameters and macular thickness were obtained using an SD Cirrus ${ }^{\mathrm{TM}}$ HD-OCT system (Version 5.0.0.326, Carl Zeiss Meditec, Dublin, CA, USA), without cycloplegia. All of the scans were performed by a single experienced examiner (I.B.-G.).

Optic disc cube $200 \times 200$ protocol was utilized to assess RNFL thickness (average and all four quadrants: superior, nasal inferior and temporal) and $\mathrm{ONH}$ measurements. This protocol generates $200 \times 200$ cube images with 200 linear scans that are performed by 200 A-scans. Cirrus ${ }^{\mathrm{TM}}$ HD-OCT software algorithms automatically detect the center of the optic disc and place a calculation circle of $3.46 \mathrm{~mm}$ diameter evenly around it. In each series of scans, average RNFL thickness and RNFL thickness in each quadrant (superior, inferior, temporal and nasal) were analyzed. ${ }^{4,18}$ The macular images were obtained using a macular cube $512 \times 128$ protocol. This protocol produces 128 horizontal scans at high resolution (512 A-scans per B-scan). RT was calculated using the built-in Macular Analysis software on the Cirrus device, which is automatically determined by taking the difference between the inner limiting membrane and the retinal pigment epithelium and provides average RT of nine zones including a $1 \mathrm{~mm}$ central zone and average macular thickness over a $6 \mathrm{~mm}$ scan diameter. Macular volume is determined on the basis of the radius of the circle subtended by the scan lines. The total macular volume corresponds to the sum of the 
volumes of the neural retina in the central $6 \mathrm{~mm}$ of the macula. ${ }^{19}$ The technique of OCT, ${ }^{2,20,21}$ as well as its reproducibility in children, ${ }^{18}$ has already been described in several reports. For image acquisition, an internal fixation target was used to ensure proper alignment of the eye. Three sequential measurements were taken, and the best centered one with signal strength of $\geq 7$ was chosen for analysis. Scan quality was checked for every OCT image, and manual correction of the boundary detection was enabled if segmentation errors were present. If an involuntary saccadic eye movement was detected during the scan, it was discarded and repeated. ${ }^{18,21}$

\subsection{Correction for ocular magnification}

Cirrus HD-OCT provides printout values of retinal features by taking default AL and refraction, which are set to $x_{0}=24.46 \mathrm{~mm}$ and $0 \mathrm{D}$, respectively. However, deviations of those previously defined parameters in the eye that is being scanned can lead to significant variations in the magnification. Littmann ${ }^{22}$ showed that the true length of a transverse retinal measurement can be derived as $t=p \cdot q \cdot s$, where $p$ is the camera constant related to the OCT imaging system, $q$ is a factor that depends on the opto-geometrical characteristics of the given eye and $s$ is the size of the retinal feature taken from the fundus image. Specifically, the factor $q$ may be estimated by the formula $q=(x-1.82 \mathrm{~mm}) \cdot 0.01306^{\circ} / \mathrm{mm}^{2}$, which depends on the $\mathrm{AL}(x)$, but neglects further rectifications caused by refractive power. ${ }^{23,24}$ As a consequence, the OCT reading $t_{0}$ will be corrected in order to achieve the true size $t=M \cdot t_{0}$, where the correction $M=(x-1.82 \mathrm{~mm}) /(24.46 \mathrm{~mm}-$ $1.82 \mathrm{~mm}$ ). As on-axis measurements of the optical instrument will not be corrected, areas taken from B-scans follow the same correction factor $M$. Finally, areas inferred from en-face images undergo a two-fold correction that is applied by means of the factor $M^{2}$. The latter also occurs for volumes.

The correction for magnification effects on the transverse scale of the OCT data, which is based on the Littman's formula, uses a factor $q$ taken from schematic eyes that are based on prototypal adult ocular dimensions. However, the accurate application of the $q$ factor to scale retinal image sizes on a child population should be subject to a reevaluation that, to the authors' knowledge, has not been reported in the literature so far. In fact, such analysis deserves a comprehensive treatment in virtue of its own relevance that is out of the scope of the present study. Accordingly, the results presented here, founded on the invariance of the factor $q$, should be considered as an advantageous approach to the unconditionally prerequisite to rectify pediatric OCT data.

\subsection{Statistical analysis}

Statistical analysis was carried out with the commercially available statistical package SPSS version 22.0 (SPSS, Chicago). Kolmogorov-Smirnov tests were used to assess sample distribution, and all variables were normally distributed. Analysis of variance (ANOVA) was used to investigate differences in mean values among the groups of children classified by $\mathrm{AL}$ and SE. To determine pairwise differences, a Bonferroni post hoc analysis was conducted when the groups were of equal sizes, and the Games-Howell post hoc test was used when the group sizes were unequal. Multiple linear regression model was constructed with RNFL thickness, RNFL thickness in the four quadrants, rim area, disc area and average cup-to-disc ratio as the dependent variables, and AL, SE and age as covariates. We also built multiple linear regression model with average macular thickness, central macular thickness and macular volume as the dependent variables and $\mathrm{AL}$ and $\mathrm{SE}$ as covariates. $\mathrm{AL}$ and $\mathrm{SE}$ were analyzed separately as they were highly correlated $(r=0.86)$. All linear regression models were examined for the presence of multicollinearity (variance inflation factor and tolerance statistics). Likewise, the independence of error assumption was assessed with the Durbin-Watson test. Residuals were ensured to be normally distributed. All data were found to exhibit homoscedasticity by assessing the plots of standardized predicted values vs studentized residuals. The presence of any significantly influential case was assessed by Cook and Mahalanobis distances. A value of $p<0.05$ was considered statistically significant.

\section{Results}

The entire group was composed of 293 eyes from 293 subjects. The mean age $( \pm \mathrm{SD})$ was $10.8 \pm 3.1$ years (range, $6-17$ years). Mean $( \pm \mathrm{SD}) \mathrm{SE}$ and $\mathrm{AL}$ 
of the whole sample were $+0.14 \pm 3.41 \mathrm{D}$ (range, -8.75 to $+8.25 \mathrm{D}$ ) and $23.12 \pm 1.49 \mathrm{~mm}$ (range, 20.24-27.24 mm), respectively. All parameters were normally distributed.

\subsection{Analysis stratified by $S E$}

The mean $( \pm \mathrm{SD})$ of descriptive and OCT parameters obtained from the entire sample and in the control, myopic and hyperopic groups are shown in Table 1(a), as well as the comparisons among them. Table 1(b) shows post hoc analysis to determine pairwise differences among groups classified according to SE refraction.

Statistically significant differences in average RNFL were found between control and myopic groups ( $p=0.002$, Bonferroni post hoc test) and between myopic and hyperopic groups $(p<0.001)$. We also obtained statistically significant differences in inferior RNFL thickness between control and myopic groups $(p<0.001)$ and between myopic and hyperopic groups $(p<0.001)$ as well as in nasal RNFL thickness between control and myopic groups $(p=0.003)$ and between myopic and hyperopic groups $(p<0.001)$. Regarding the macular parameters, statistically significant differences in the three parameters evaluated among the three groups assessed (average macular thickness, central macular thickness and macular volume) were found, as shown in Table 1(a).

Table 2(a) summarizes descriptive and OCT parameters in the control group and in the myopic and hyperopic groups subdivided into three refractive subgroups and the comparisons among them. Tables 2(b)-2(e) show post hoc analysis to determine pairwise differences among groups classified according to SE refraction subgroups (GamesHowell post hoc analysis). It should be observed that we did not include rim area, disc area and RNFL in temporal quadrant in these tables, because we did not find pairwise differences among them.

Statistically significant differences in average RNFL thickness were found between high myopic subgroup compared with low myopic subgroup, control group and low, moderate and high hyperopic subgroups $(p<0.05$ for all comparisons, GamesHowell post hoc test). No significant differences between high and moderate myopic subgroups were found. We also found statistically significant differences in superior and inferior RNFL thickness between high myopic subgroup compared with all subgroups analyzed ( $p<0.05$ for all comparisons), except for the moderate myopic one. Concerning the nasal RNFL thickness, statistically significant differences were found between high myopic subgroup compared with control group and moderate and high hyperopic subgroups ( $p<0.05$ for all comparisons). Statistically significant differences in cup-to-disc ratio $(C / D)$ between high myopic subgroup compared with high hyperopic subgroup $(p<0.05)$ were obtained. No significant differences between other diagnostic subgroups were found (Tables 2(b)-2(e)).

The results also revealed statistically significant differences in average macular thickness between high myopic subgroup compared with moderate

Table 1a. Descriptive and OCT parameters in the entire group and in the three groups examined: emmetropic, myopic and hyperopic groups.

\begin{tabular}{|c|c|c|c|c|c|}
\hline & $\begin{array}{l}\text { All subjects } \\
(n=293)\end{array}$ & $\begin{array}{c}\text { Myopic } \\
(n=100)\end{array}$ & $\begin{array}{l}\text { Emmetropic } \\
\quad(n=99)\end{array}$ & $\begin{array}{c}\text { Hyperopic } \\
(n=94)\end{array}$ & $\begin{array}{c}p \text { (by one-way } \\
\text { ANOVA) }\end{array}$ \\
\hline Age (years) & $10.84 \pm 3.05$ & $12.11 \pm 2.76$ & $11.88 \pm 2.97$ & $9.08 \pm 2.57$ & $<0.001^{*}$ \\
\hline Axial length (mm) & $23.12 \pm 1.49$ & $24.51 \pm 1.11$ & $23.12 \pm 0.79$ & $21.64 \pm 0.89$ & $<0.001^{*}$ \\
\hline SE refraction (D) & $+0.14 \pm 0.51$ & $-3.32 \pm 2.32$ & $+0.34 \pm 0.41$ & $+3.99 \pm 1.82$ & $<0.001^{*}$ \\
\hline Average RNFL $(\mu \mathrm{m})$ & $99.46 \pm 11.21$ & $95.20 \pm 10.04$ & $100.39 \pm 11.31$ & $103.02 \pm 10.91$ & $<0.001^{*}$ \\
\hline Superior RNFL ( $\mu \mathrm{m})$ & $123.63 \pm 22.76$ & $121.89 \pm 25.00$ & $123.53 \pm 23.81$ & $125.60 \pm 18.90$ & 0.49 \\
\hline Temporal RNFL $(\mu \mathrm{m})$ & $72.73 \pm 16.33$ & $72.47 \pm 15.98$ & $74.51 \pm 17.78$ & $71.15 \pm 15.07$ & 0.35 \\
\hline Inferior RNFL $(\mu \mathrm{m})$ & $125.75 \pm 23.01$ & $116.70 \pm 17.81$ & $130.11 \pm 21.33$ & $130.82 \pm 26.64$ & $<0.001^{*}$ \\
\hline Nasal RNFL $(\mu \mathrm{m})$ & $70.20 \pm 15.07$ & $65.16 \pm 12.29$ & $72.12 \pm 16.77$ & $73.53 \pm 14.63$ & $<0.001^{*}$ \\
\hline $\operatorname{Rim}$ area $\left(\mathrm{mm}^{2}\right)$ & $1.56 \pm 0.29$ & $1.58 \pm 0.28$ & $1.55 \pm 0.30$ & $1.54 \pm 0.31$ & 0.60 \\
\hline Disc area $\left(\mathrm{mm}^{2}\right)$ & $1.88 \pm 0.34$ & $1.85 \pm 0.34$ & $1.94 \pm 0.42$ & $1.84 \pm 0.30$ & 0.06 \\
\hline Cup-to-disc ratio $(C / D)$ & $0.34 \pm 0.19$ & $0.35 \pm 0.19$ & $0.36 \pm 0.17$ & $0.31 \pm 0.18$ & 0.08 \\
\hline Average macular thickness $(\mu \mathrm{m})$ & $281.94 \pm 21.45$ & $275.52 \pm 30.65$ & $282.90 \pm 12.37$ & $287.74 \pm 14.42$ & $0.002^{*}$ \\
\hline Central macular thickness $(\mu \mathrm{m})$ & $253.60 \pm 19.80$ & $255.56 \pm 20.05$ & $259.21 \pm 19.30$ & $245.53 \pm 17.40$ & $<0.001^{*}$ \\
\hline Macular volume $\left(\mathrm{mm}^{3}\right)$ & $9.56 \pm 0.69$ & $9.06 \pm 0.63$ & $9.57 \pm 0.53$ & $10.02 \pm 0.64$ & $<0.001^{*}$ \\
\hline
\end{tabular}

Note: ${ }^{*}$ Statistically significant. 
Table 1b. Post hoc analysis to determine pairwise differences among groups classified according to SE refraction (Bonferroni post hoc analysis).

\begin{tabular}{|c|c|c|c|}
\hline & Comparative groul & to SE refraction & $p$-value \\
\hline \multirow[t]{3}{*}{ Average RNFL $(\mu \mathrm{m})$} & Emmetropic & Myopic & $0.002^{*}$ \\
\hline & Emmetropic & Hyperopic & 0.36 \\
\hline & Myopic & Hyperopic & $<0.001^{*}$ \\
\hline \multirow[t]{3}{*}{ Superior RNFL $(\mu \mathrm{m})$} & Emmetropic & Myopic & 0.90 \\
\hline & Emmetropic & Hyperopic & 0.88 \\
\hline & Myopic & Hyperopic & 0.77 \\
\hline \multirow[t]{3}{*}{ Temporal RNFL $(\mu \mathrm{m})$} & Emmetropic & Myopic & 0.89 \\
\hline & Emmetropic & Hyperopic & 0.44 \\
\hline & Myopic & Hyperopic & 0.75 \\
\hline \multirow[t]{3}{*}{ Inferior RNFL $(\mu \mathrm{m})$} & Emmetropic & Myopic & $<0.001^{*}$ \\
\hline & Emmetropic & Hyperopic & 0.68 \\
\hline & Myopic & Hyperopic & $<0.001^{*}$ \\
\hline \multirow[t]{3}{*}{ Nasal RNFL $(\mu \mathrm{m})$} & Emmetropic & Myopic & $0.003^{*}$ \\
\hline & Emmetropic & Hyperopic & 0.72 \\
\hline & Myopic & Hyperopic & $<0.001^{*}$ \\
\hline \multirow[t]{3}{*}{ Rim area $\left(\mathrm{mm}^{2}\right)$} & Emmetropic & Myopic & 0.90 \\
\hline & Emmetropic & Hyperopic & 0.88 \\
\hline & Myopic & Hyperopic & 0.85 \\
\hline \multirow[t]{3}{*}{ Disc area $\left(\mathrm{mm}^{2}\right)$} & Emmetropic & Myopic & 0.13 \\
\hline & Emmetropic & Hyperopic & 0.09 \\
\hline & Myopic & Hyperopic & 0.11 \\
\hline \multirow[t]{3}{*}{ Cup-to-disc ratio $(C / D)$} & Emmetropic & Myopic & $0.03^{*}$ \\
\hline & Emmetropic & Hyperopic & 0.05 \\
\hline & Myopic & Hyperopic & $0.02 *$ \\
\hline \multirow[t]{3}{*}{ Average macular thickness $(\mu \mathrm{m})$} & Emmetropic & Myopic & $0.04^{*}$ \\
\hline & Emmetropic & Hyperopic & 0.30 \\
\hline & Myopic & Hyperopic & $<0.001^{*}$ \\
\hline \multirow[t]{3}{*}{ Central macular thickness $(\mu \mathrm{m})$} & Emmetropic & Myopic & 0.56 \\
\hline & Emmetropic & Hyperopic & $0.001^{*}$ \\
\hline & Myopic & Hyperopic & $<0.001^{*}$ \\
\hline \multirow[t]{3}{*}{ Macular volume $\left(\mathrm{mm}^{3}\right)$} & Emmetropic & Myopic & $<0.001^{*}$ \\
\hline & Emmetropic & Hyperopic & $<0.001^{*}$ \\
\hline & Myopic & Hyperopic & $<0.001^{*}$ \\
\hline
\end{tabular}

Note: *Statistically significant.

and high hyperopic subgroups $(p<0.05$ for all comparisons, Games-Howell post hoc test), as well as in central macular thickness between high myopic subgroup compared with moderate and high hyperopic subgroups ( $p<0.05$ for all comparisons). Statistically significant differences in macular volume between high myopic subgroup compared with all subgroups analyzed were found $(p<0.05$ for all comparisons), except for the moderate myopic one (Tables 2(b)-2(e)).

\subsection{Analysis stratified by $A L$}

Table 3(a) shows descriptive and OCT parameters in the three groups examined according to AL: short ( $<22.00 \mathrm{~mm}, 33$ boys and 35 girls), medium (from $\geq 22.00 \mathrm{~mm}$ to $25.00 \mathrm{~mm}, 88$ boys and 101 girls) and long eyes ( $>25.00 \mathrm{~mm}, 19$ boys and 17 girls) and the comparisons between them. Table $3(\mathrm{~b})$ shows post hoc analysis to determine pairwise differences among groups classified according to AL.

Statistically significant differences in average RNFL thickness and in the thicknesses in inferior and nasal quadrants were found between short eyes group compared with medium and long eyes groups and between medium and long eyes groups $(p<0.05$ for all comparisons, Games-Howell post hoc test). There were statistically significant differences in average macular thickness, central macular thickness and macular volume among short eyes group compared with medium and long eyes groups ( $p<0.05$ for all comparisons). 
OCT measurements in children
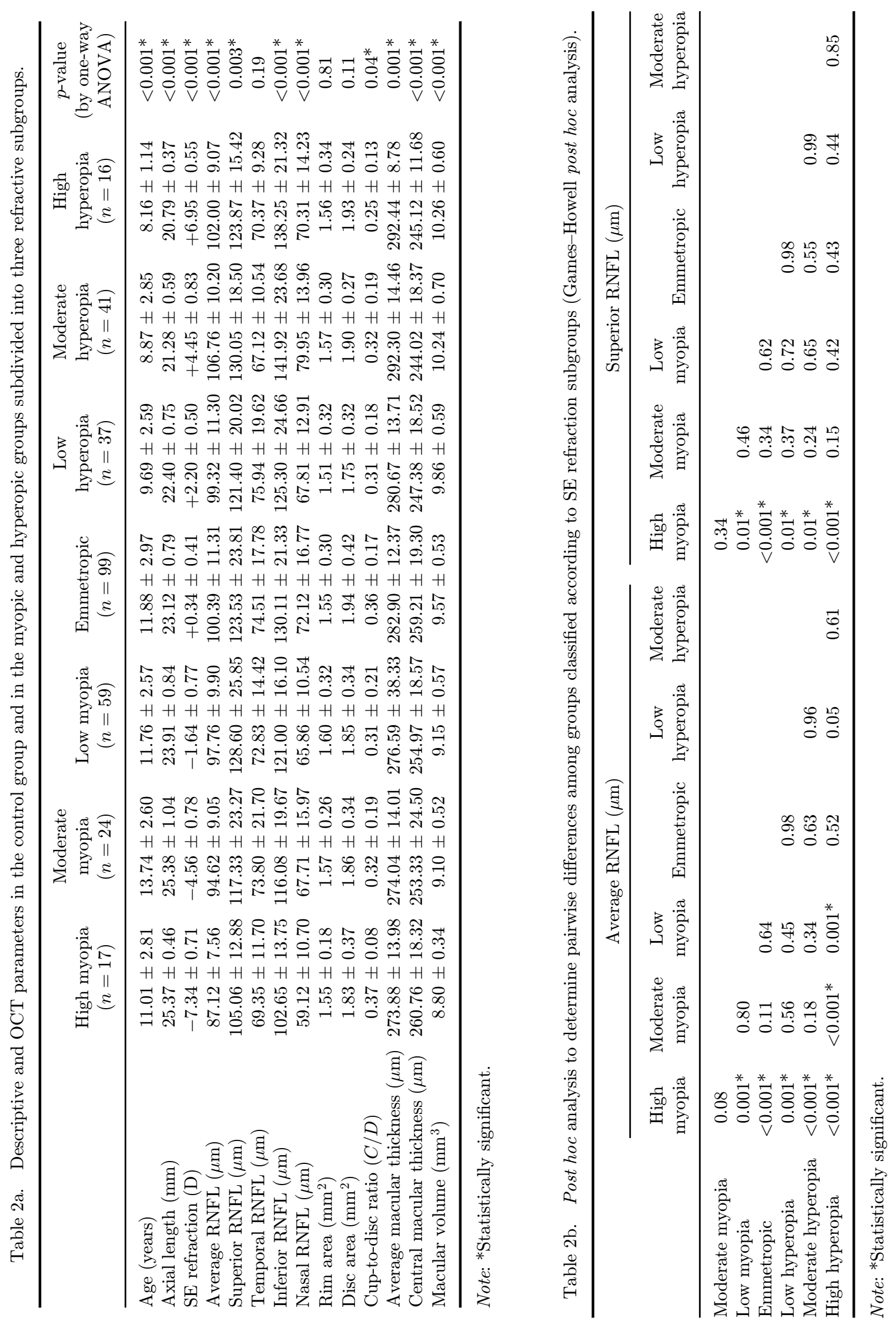


\section{Bueno-Gimeno et al.}

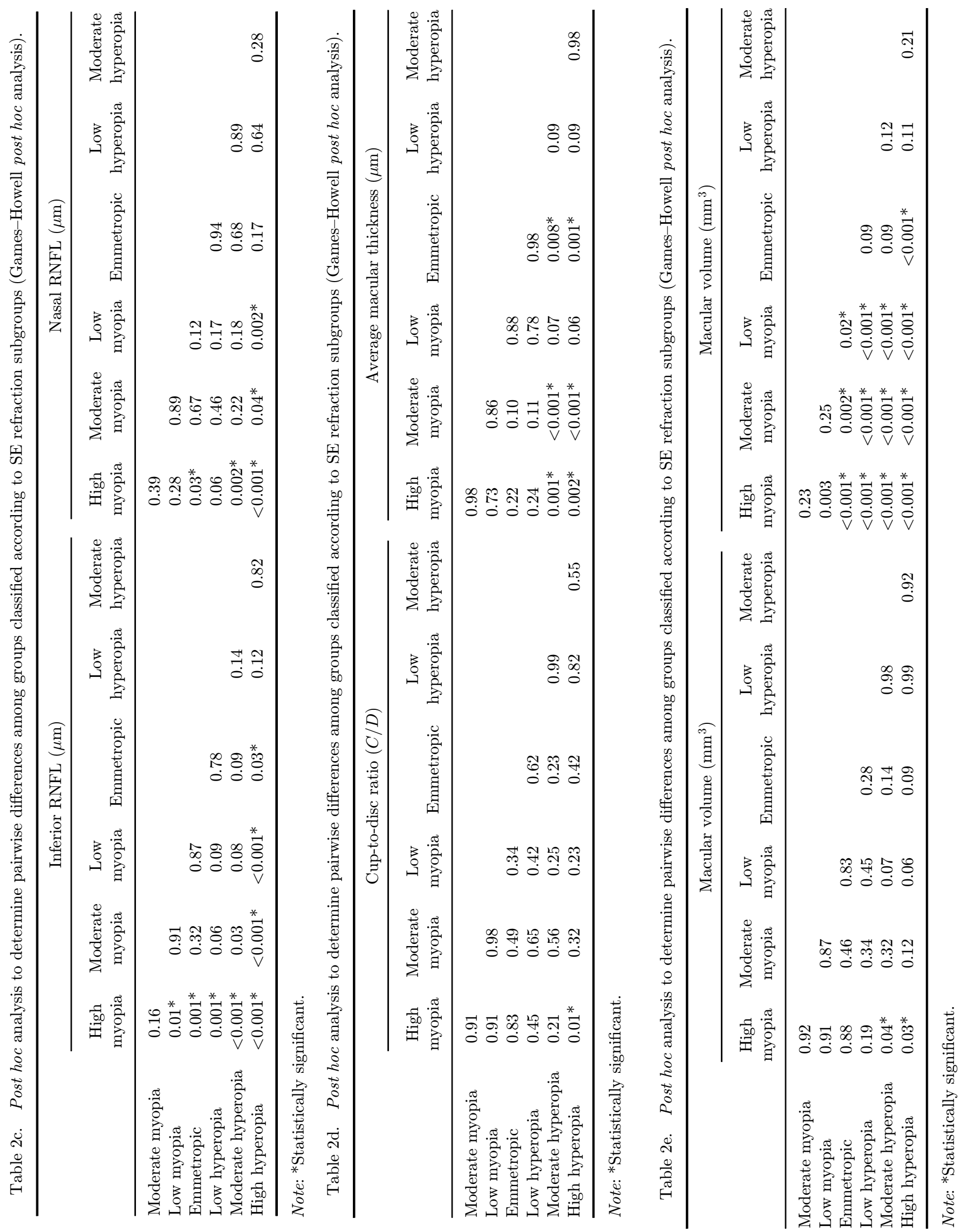


Table 3a. Descriptive and OCT parameters in the three groups examined according to AL: short, medium and long eyes.

\begin{tabular}{|c|c|c|c|c|}
\hline & $\begin{array}{l}\text { Axial length } \\
<22.00(\mathrm{~mm}) \\
n=68\end{array}$ & $\begin{array}{c}\text { Axial length } \\
\geq 22.00 \text { to } 25.00(\mathrm{~mm}) \\
n=189\end{array}$ & $\begin{array}{c}\text { Axial length } \\
>25.00(\mathrm{~mm}) \\
n=36\end{array}$ & $\begin{array}{c}p \text {-value } \\
\text { (by one-way } \\
\text { ANOVA) }\end{array}$ \\
\hline Age (years) & $8.48 \pm 2.46$ & $11.37 \pm 2.86$ & $12.51 \pm 2.70$ & $<0.001^{*}$ \\
\hline Axial length $(\mathrm{mm})$ & $21.15 \pm 0.47$ & $23.35 \pm 0.81$ & $25.67 \pm 0.54$ & $<0.001^{*}$ \\
\hline SE refraction (D) & $+4.24 \pm 2.19$ & $-0.30 \pm 1.92$ & $-5.30 \pm 2.18$ & $<0.001^{*}$ \\
\hline Average RNFL $(\mu \mathrm{m})$ & $104.78 \pm 10.59$ & $98.75 \pm 10.85$ & $93.17 \pm 10.19$ & $<0.001^{*}$ \\
\hline Superior RNFL $(\mu \mathrm{m})$ & $126.31 \pm 17.56$ & $124.56 \pm 24.16$ & $113.72 \pm 21.88$ & 0.17 \\
\hline Temporal RNFL $(\mu \mathrm{m})$ & $69.62 \pm 15.35$ & $73.45 \pm 16.16$ & $74.83 \pm 18.60$ & 0.18 \\
\hline Inferior RNFL $(\mu \mathrm{m})$ & $137.40 \pm 22.54$ & $124.05 \pm 21.67$ & $112.61 \pm 21.55$ & $<0.001^{*}$ \\
\hline Nasal RNFL $(\mu \mathrm{m})$ & $76.26 \pm 14.61$ & $68.95 \pm 14.76$ & $65.22 \pm 14.49$ & $<0.001^{*}$ \\
\hline $\operatorname{Rim}$ area $\left(\mathrm{mm}^{2}\right)$ & $1.55 \pm 0.30$ & $1.56 \pm 0.29$ & $1.61 \pm 0.26$ & 0.46 \\
\hline Disc area $\left(\mathrm{mm}^{2}\right)$ & $1.83 \pm 0.28$ & $1.89 \pm 0.33$ & $1.94 \pm 0.45$ & 0.28 \\
\hline Cup-to-disc ratio $(C / D)$ & $0.28 \pm 0.17$ & $0.34 \pm 0.19$ & $0.33 \pm 0.18$ & $0.01^{*}$ \\
\hline Average macular thickness $(\mu \mathrm{m})$ & $288.87 \pm 13.97$ & $280.90 \pm 24.11$ & $274.36 \pm 13.52$ & $0.002^{*}$ \\
\hline Central macular thickness $(\mu \mathrm{m})$ & $243.33 \pm 15.93$ & $256.40 \pm 19.57$ & $258.17 \pm 21.08$ & $<0.001^{*}$ \\
\hline Macular volume $\left(\mathrm{mm}^{3}\right)$ & $10.40 \pm 0.60$ & $9.65 \pm 0.54$ & $8.87 \pm 0.44$ & $<0.001^{*}$ \\
\hline
\end{tabular}

Note: *Statistically significant.

Table 3b. Post hoc analysis to determine pairwise differences among groups classified according to AL (Games-Howell post hoc analysis).

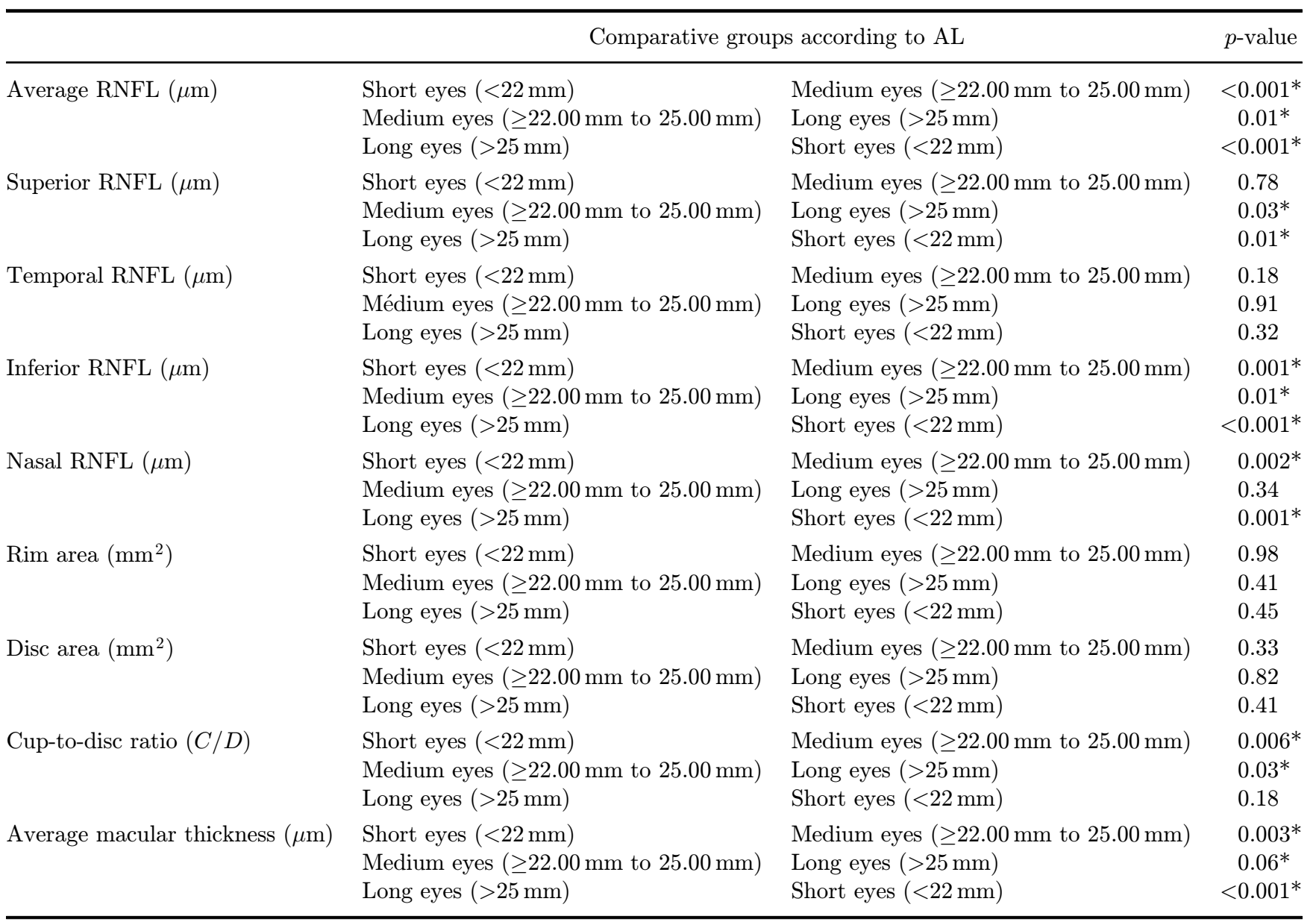


Table 3b. (Continued)

\begin{tabular}{llll}
\hline & & Comparative groups according to AL & $p$-value \\
\hline Central macular thickness $(\mu \mathrm{m})$ & Short eyes $(<22 \mathrm{~mm})$ & Medium eyes $(\geq 22.00 \mathrm{~mm}$ to $25.00 \mathrm{~mm})$ & $<0.001^{*}$ \\
& Medium eyes $(\geq 22.00 \mathrm{~mm}$ to $25.00 \mathrm{~mm})$ & Long eyes $(>25 \mathrm{~mm})$ & 0.18 \\
& Long eyes $(>25 \mathrm{~mm})$ & Short eyes $(<22 \mathrm{~mm})$ & $0.001^{*}$ \\
Macular volume $\left(\mathrm{mm}^{3}\right)$ & Short eyes $(<22 \mathrm{~mm})$ & Medium eyes $(\geq 22.00 \mathrm{~mm} \mathrm{to} 25.00 \mathrm{~mm}) \quad<0.001^{*}$ \\
& Medium eyes $(\geq 22.00 \mathrm{~mm}$ to $25.00 \mathrm{~mm})$ & Long eyes $(>25 \mathrm{~mm})$ & Short eyes $(<22 \mathrm{~mm})$
\end{tabular}

Note: *Statistically significant.

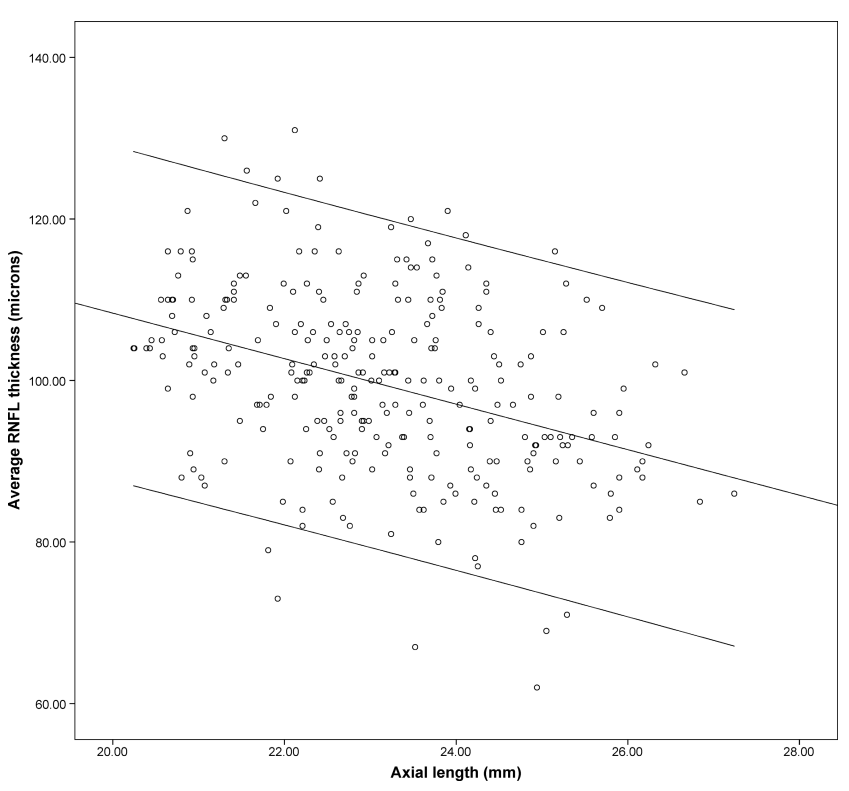

Fig. 1. Correlation between average RNFL thickness $(\mathrm{m} \mu)$ and $\mathrm{AL}(\mathrm{mm})$.

\subsection{Linear regression analysis}

Average RNFL thickness (Fig. 1) and the thickness in superior, inferior and nasal quadrants were shown to decrease as the AL increases. Linear regression revealed significant negative correlations of AL with average RNFL thickness and thicknesses in superior, inferior and nasal quadrants. Consistent relationships of SE with average RNFL thickness and thicknesses in superior, inferior and nasal quadrants were also found. These associations are described in Table 4.

Table 5 displays the results of the linear regression analysis of the relationship of AL and SE with macular parameters. AL correlated negatively with average macular thickness (Fig. 2) and macular volume and positively with central macular thickness. In contrast, negative SE was also negatively associated with average macular thickness and macular volume and positively with central macular thickness.

Table 4. Results of multivariate-mixed model analysis of the association between AL, SE and age and RNFL thickness, RNFL thickness in the four quadrants, rim area, disc area and average cup-to disc-ratio.

\begin{tabular}{|c|c|c|c|c|c|}
\hline & Beta & Regression coefficient $(95 \% \mathrm{CI})$ & Standard error & $p$-value & $F$-value \\
\hline \multicolumn{6}{|l|}{ Axial length (mm) } \\
\hline Average RNFL $(\mu \mathrm{m})$ & -0.56 & $-2.96(-3.86$ to -2.07$)$ & 0.45 & $<0.001^{*}$ & 8.12 \\
\hline Superior RNFL $(\mu \mathrm{m})$ & 0.28 & $-2.85(-4.78$ to -0.93$)$ & 0.98 & $0.004^{*}$ & 0.18 \\
\hline Temporal RNFL $(\mu \mathrm{m})$ & 0.18 & $0.96(-0.43$ to 2.35$)$ & 0.70 & 0.17 & 0.15 \\
\hline Inferior RNFL $(\mu \mathrm{m})$ & -0.04 & $-5.65(-7.51$ to -3.78$)$ & 0.85 & $<0.001^{*}$ & 0.52 \\
\hline Nasal RNFL $(\mu \mathrm{m})$ & 0.01 & $-3.37(-4.60$ to -2.13$)$ & 0.60 & $<0.001^{*}$ & 4.64 \\
\hline $\operatorname{Rim}$ area $\left(\mathrm{mm}^{2}\right)$ & 0.28 & $0.01(-0.01$ to 0.40$)$ & 0.01 & 0.34 & 2.97 \\
\hline Disc area $\left(\mathrm{mm}^{2}\right)$ & -0.05 & $0.01(-0.02$ to 0.04$)$ & 0.01 & 0.65 & 8.75 \\
\hline Cup-to-disc ratio $(C / D)$ & 0.17 & $0.01(-0.01$ to 0.01$)$ & 0.01 & 0.31 & 0.70 \\
\hline \multicolumn{6}{|l|}{$\mathrm{SE}$ refraction (D) } \\
\hline Average RNFL $(\mu \mathrm{m})$ & 0.35 & $-1.15(-1.55$ to -0.76$)$ & 0.19 & $<0.001^{*}$ & 0.84 \\
\hline Superior RNFL $(\mu \mathrm{m})$ & -0.08 & $-1.35(-1.90$ to -0.40$)$ & 0.42 & $0.001^{*}$ & 2.10 \\
\hline Temporal RNFL $(\mu \mathrm{m})$ & -0.15 & $0.29(0.32$ to -0.91$)$ & 0.30 & 0.53 & 1.90 \\
\hline
\end{tabular}


Table 4. (Continued)

\begin{tabular}{lrccrr}
\hline & Beta & Regression coefficient $(95 \%$ CI $)$ & Standard error & $p$-value & $F$-value \\
\hline Inferior RNFL $(\mu \mathrm{m})$ & 0.15 & $-2.72(-3.50$ to -1.93$)$ & 0.40 & $<0.001^{*}$ & 10.12 \\
Nasal RNFL $(\mu \mathrm{m})$ & 0.01 & $-1.35(-1.88$ to -0.82$)$ & 0.27 & $<0.001^{*}$ & 0.99 \\
Rim area $\left(\mathrm{mm}^{2}\right)$ & -0.33 & $-0.03(-0.01$ to 0.01$)$ & 0.05 & 0.85 & 2.06 \\
Disc area $\left(\mathrm{mm}^{2}\right)$ & 0.20 & $0.01(1.88$ to -1.97$)$ & 0.06 & 0.14 & 10.79 \\
Cup-to-disc ratio $(C / D)$ & -0.24 & $-0.02(-0.08$ to 0.06$)$ & 0.03 & 0.74 & 0.33 \\
Age (years) & & & & & \\
Average RNFL $(\mu \mathrm{m})$ & -0.45 & $-0.12(-0.19$ to -0.05$)$ & 0.22 & 0.42 & 0.66 \\
Superior RNFL $(\mu \mathrm{m})$ & 0.23 & $0.03(0.10$ to -0.05$)$ & 0.48 & 0.07 & 3.57 \\
Temporal RNFL $(\mu \mathrm{m})$ & 0.04 & $0.01(-0.2$ to 0.03$)$ & 0.35 & 0.06 & 5.47 \\
Inferior RNFL $(\mu \mathrm{mm})$ & 0.08 & $0.01(-0.01$ to 0.03$)$ & 0.46 & 0.08 & 3.90 \\
Nasal RNFL $(\mu \mathrm{m})$ & 0.17 & $0.03(0.01$ to -0.06$)$ & 0.31 & 0.08 & 8.21 \\
Rim area $\left(\mathrm{mm}{ }^{2}\right)$ & 0.15 & $0.42(-0.84$ to 3.95$)$ & 0.01 & 0.36 & 0.84 \\
Disc area $\left(\mathrm{mm}^{2}\right)$ & 0.05 & $0.43(-1.51$ to 2.37$)$ & 0.01 & 0.06 & 4.21 \\
Cup-to-disc ratio $(C / D)$ & 0.11 & $1.86(-1.55$ to 5.26$)$ & 0.04 & 0.30 & 1.10 \\
\hline
\end{tabular}

Note: *Statistically significant. CI, confidence interval.

Table 5. Results of multivariate-mixed model analysis of the association between AL, SE and age and average macular thickness, central macular thickness and macular volume.

\begin{tabular}{|c|c|c|c|c|c|}
\hline & Beta & Regression coefficient (95\% CI) & Standard error & $p$-value & $F$-value \\
\hline \multicolumn{6}{|l|}{ Axial length (mm) } \\
\hline Average macular thickness $(\mu \mathrm{m})$ & -0.42 & $-3.24(-5.05$ to -1.43$)$ & 0.92 & $0.001^{*}$ & 0.56 \\
\hline Central macular thickness $(\mu \mathrm{m})$ & -0.43 & $-0.03(-0.10$ to -0.00$)$ & 0.02 & $<0.001^{*}$ & 0.30 \\
\hline Macular volume $\left(\mathrm{mm}^{3}\right)$ & -0.08 & $-1.91(-2.06$ to -1.75$)$ & 0.03 & $<0.001^{*}$ & 17.09 \\
\hline \multicolumn{6}{|l|}{ SE refraction $(\mathrm{D})$} \\
\hline Average macular thickness $(\mu \mathrm{m})$ & 0.45 & $-1.86(-2.63$ to -1.04$)$ & 0.38 & $<0.001^{*}$ & 10.90 \\
\hline Central macular thickness $(\mu \mathrm{m})$ & 0.04 & $-0.71(-1.41$ to -0.08$)$ & 0.39 & $<0.001^{*}$ & 0.44 \\
\hline Macular volume $\left(\mathrm{mm}^{3}\right)$ & -0.04 & $-0.11(-0.13$ to -0.09$)$ & 0.03 & $<0.001^{*}$ & 6.70 \\
\hline \multicolumn{6}{|l|}{ Age (years) } \\
\hline Average macular thickness $(\mu \mathrm{m})$ & 0.32 & $0.71(-0.14$ to 0.15$)$ & 0.28 & 0.19 & 2.10 \\
\hline Central macular thickness $(\mu \mathrm{m})$ & 0.16 & $1.41(0.63$ to -2.19$)$ & 0.39 & $0.007^{*}$ & 11.26 \\
\hline Macular volume $\left(\mathrm{mm}^{3}\right)$ & 0.32 & $1.43(0.90$ to -1.95$)$ & 0.01 & $<0.001^{*}$ & 0.43 \\
\hline
\end{tabular}

Note: *Statistically significant. CI, confidence interval.

\section{Discussion}

In the present study, we investigated the impact of $\mathrm{AL}$ and refractive error on OCT measurements taking into account the effect of ocular magnification for transverse measurements. Elía et $a l^{25}$ asserted that related ocular magnification had a minimal effect on RNFL and macular thickness measurements. In their work, refractive errors ranged from $-2.50 \mathrm{D}$ to $+6.25 \mathrm{D}$ and did not measure AL. However, we considered a suitable correction for ocular magnification because Cirrus HDOCT (Carl Zeiss Meditec) provides printout values of retinal features assuming default AL and refraction, which are set to $x_{0}=24.46 \mathrm{~mm}$ and $0 \mathrm{D}$, respectively. However, significant variations in the magnification applied to the fundus image are evident, provided that AL and refraction (the latter leading to minimal changes) of the evaluated eye deviate from that considered by default. For this reason, some studies carried out in adults ${ }^{4}$ and children $^{2,6,8}$ corrected OCT measurements for ocular magnification, similar to our study. It should be mentioned that the Littmann formula employed in our analysis for the correction of retina-related sizes normally to the optic axis cannot be directly applied to a given retinal layer thickness, which constitutes an erroneous procedure followed in numerous studies. Note that a measurement of any layer 


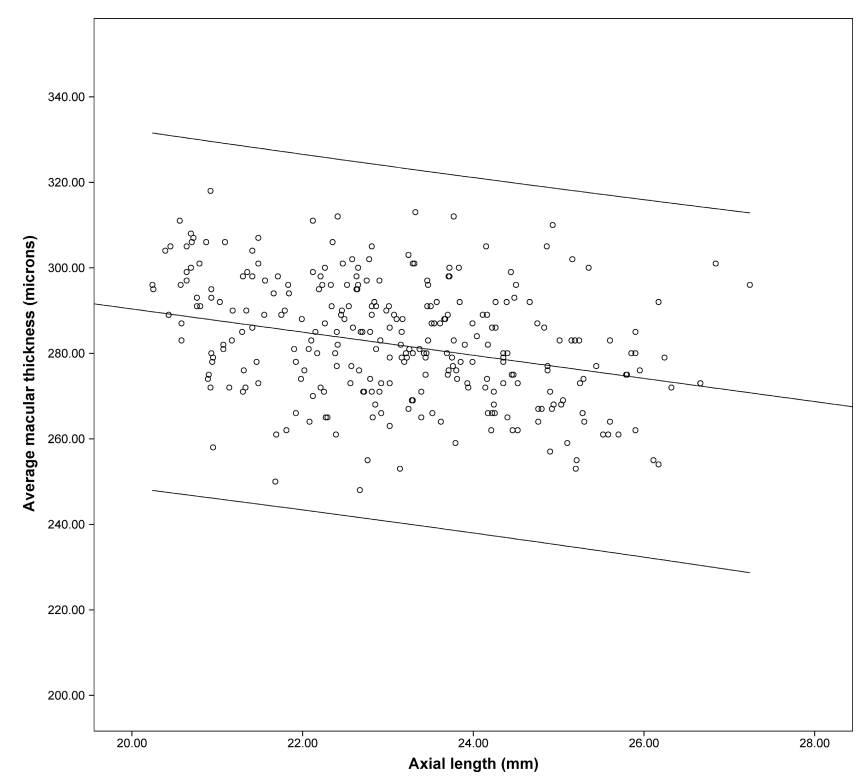

Fig. 2. Correlation between average macular thickness $(\mathrm{m} \mu)$ and $\mathrm{AL}(\mathrm{mm})$.

depth is governed by the source coherence, which is unaltered by the geometrical magnification inherent to the formation of fundus images.

Our findings showed that average RNFL thickness of all quadrants, except for the temporal one, were thinner in myopic eyes when compared with hyperopic and emmetropic eyes. On the other hand, we did not find statistically significant differences among the three groups evaluated in the rim area and disc area. However, we found statistically significant differences between control and myopic groups and between myopic and hyperopic groups in cup-to-disc ratio. When we compared pairwise differences among the groups classified according to AL, we did not find statistical significance in rim and disc area parameters.

We also categorized the myopic and hyperopic groups into subgroups according to $\mathrm{SE}$ and obtained that the average RNFL thickness and the RNFL thickness in the superior, inferior and nasal quadrants were thinner in high myopic subgroup compared with low myopic subgroup, control group and low, moderate and high hyperopic subgroups. With reference to rim and disc area, our results did not show statistically significant differences in post $h o c$ analysis. These results are in agreement with the results of earlier studies carried out in both adults $^{26}$ and children, ${ }^{8}$ which revealed significantly lower values of average peripapillary RNFL thickness in high myopic subjects. In Caucasian children,
Barrio-Barrio et al. ${ }^{27}$ found a significant association between RNFL (without correction for magnification) and SE and a tendency toward significance between AL and RNFL. In our series, there were no significant differences in temporal quadrant thickness among the groups and subgroups evaluated. This would be in agreement with that given by Lim and Chun, ${ }^{8}$ who found that temporal RNFL thickness remained unaffected by increasing myopia.

Taş et $a l .{ }^{6}$ reported significant differences between low and high hyperopia groups regarding the average RNFL thickness and the RNFL thicknesses of inferior and nasal quadrants in hyperopic children. These findings also are consistent with our data. However, the authors ${ }^{6}$ found that differences among hyperopia subgroups in RNFL thickness disappeared when the magnification attributable to $\mathrm{SE} / \mathrm{AL}$ was taken into account. We also investigated the effect of AL on OCT measurements. We found that the average RNFL thickness and the thickness in the inferior and nasal quadrants decreased as AL increased. This finding is in agreement with the previous report. ${ }^{4}$ However, in the present study, associations among $\mathrm{ONH}$ parameters and AL or SE after applying the correction for ocular magnification, as other researchers reported, ${ }^{2,4}$ were not found. Huynh et al. ${ }^{2}$ referred an increasing optic disc area and a decreasing rim area with longer eyes in a sample predominantly hyperopic. Likewise, Savini et al. ${ }^{4}$ observed an inverse association between $\mathrm{AL}$ and $\mathrm{OHN}$ parameters (disc and rim areas). It should be highlighted that before applying the correction for ocular magnification, negative correlations of $\mathrm{AL}$ and negative SE with disc and rim areas were found. These associations disappeared when the correction for ocular magnification was implemented. Our results did not show correlation among disc and rim areas with $\mathrm{AL}, \mathrm{SE}$ and age, as we reported in Tables 4 and 5 .

The results of our study differ from those obtained in previous studies. We did not apply the Littmann formula to the RNFL thicknesses as it has been justified above. This could explain the disagreements between our results and those given by other investigators in studies in which the Littmann formula has been applied or has not been correctly implemented. ${ }^{4,27}$ As other authors suggested, when this formula is applied, the relationships of OCT measurements with other refractive or anatomical parameters might change, except for age. ${ }^{4,6}$ Other factors that may have accounted for differences 
between our study and previous one would be the diverse races analyzed, the number of subjects, the range of refractive error included in the study and the different devices used to take the measurements.

To our knowledge, there are no studies evaluating the effect of both AL and SE on OCT measurements, especially in Caucasian children. To this date, only two previous studies have been conducted in Spanish children evaluating OCT parameters. ${ }^{25,27}$ There are some differences between our study and these previous ones, ${ }^{25,27}$ such as the range of age, the amount of refractive error and the parameters included in the analysis. In spite of this, we obtained some results similar to those given by these authors. ${ }^{25,27}$ Although AL and SE are significantly correlated, the analysis of the effect of these parameters on OCT measurements was justified as the refractive error is the result of the balance among the refractive power of the optical elements of the eye and AL. Therefore, both parameters are not representing exactly the same.

With reference to macular parameters, the longer the eye, the thinner was the average macular thickness and the smaller macular volume. We also found thinner average macular thickness and smaller macular volume in myopic eyes compared with hyperopic and emmetropic eyes. However, central macular thickness was thinner in hyperopic eyes in comparison to myopic and emmetropic eyes. When comparing the refractive subgroups, our results showed that average macular thickness and macular volume decreased as the level of myopia increased. In contrast, we found thinner central macular thickness in shorter and more hyperopic eyes. These results are in agreement with those provided by other researchers in both adults ${ }^{28}$ and children. ${ }^{8} \mathrm{Wu}$ et al. ${ }^{28}$ reported that $\mathrm{RT}$ in individuals with high myopia and long eyes was thicker in the foveola and fovea, but thinner in the inner and the outer macular regions. These authors also showed smaller macular volume in subjects with high myopia. ${ }^{28}$ Lim and $\mathrm{Chun}^{8}$ also obtained thinner macular thickness and lower macular volumes in high myopic children. It has been suggested that these relations would be owing to the mechanical globe elongation linked with myopia, resulting in retinal stretching and thinning. ${ }^{8,29}$ This could explain the decrease in RNFL and macular thickness. Ostrin et al., ${ }^{30}$ in a study performed in adults, reported that high myopes tend to have a thinner subfoveal choroid and larger scleral canal. They referred that these relationships might help to explain the increased risk in glaucoma. Our outcomes showing RNFL thinning, average macular thinning, smaller macular volume and central macular thickening in longer and more myopic eyes support this suggestion. It should be noted that there were few highly myopic children in our sample as the population with high myopia is much reduced, especially in white children and therefore less population is required to have a relevant sample. ${ }^{12}$ Note that in our sample the mean age $( \pm \mathrm{SD})$ was $10.8 \pm 31$ as Foster and Jiang ${ }^{31}$ referred; the prevalence rates of high myopia at this age in Caucasian children are less common.

We did not find age-related changes of the RNFL thicknesses, ONH parameters and macular thickness. In contrast, a positive association was found between central macular thickness and macular volume with age. Barrio et al. ${ }^{27}$ reported positive associations between average macular thickness and macular volume with age, but they did not find correlation between RNFL and age. Huynh et al. ${ }^{29}$ also reported thinner central macular thickness in younger children. However, some previous pediatric studies have shown that RNFL thickness was not associated with age. ${ }^{25-33}$ It seems that the onset of decrease in the RFNL with age does not appear to be significant until the age of 15 years. ${ }^{33}$ We consider, as Huynh et $a l^{34}$ suggested, that the growth of macular, RNFL and optic disc parameters is almost complete at birth or soon after it. Therefore, we believe that these correlations are likely to be clinically insignificant.

In the Cirrus HD-OCT, the normative database is not available for patients under 18 years of age. Furthermore, when the database was built, the manufacturers did not take into account the effect of AL and refractive error, although several studies have shown the significant effect of both parameters on OCT measurements, ${ }^{2,4,8,26,28,32}$ regardless of age. In addition, a magnification correction is not provided by the Cirrus HD-OCT manufacturers. Kang et $a l .{ }^{9}$ proposed that the effect of ocular magnification should be taken into consideration for myopic eyes greater than $-4.00 \mathrm{D}$. Myopia is the most prevalent refractive error in the world, and the number of people with myopia continues to increase worldwide. ${ }^{35} \mathrm{~A}$ lot of research is currently being conducted to investigate causes and development of myopia. High levels of myopia are linked with ocular pathologies such as retinal detachment and 
chorioretinal degeneration risk of developing glaucoma. It is considered very important to follow up changes in retinal parameters in myopic and in glaucoma patients. This is feasible with OCT devices, but it is reported that each instrument results in variability in RT measurements, due to the algorithms used by each OCT. Therefore, we consider that the magnification on lateral measurements ${ }^{10}$ such as disc, rim area and macular volume must be corrected for establishing diagnosis and treatment protocols, specially in myopic and glaucoma patients.

Therefore, from our point of view in future improvements of the instrument or when the software is updated, the manufacturers might introduce corrections to take into account the effect of related ocular magnification and set up a new database including subjects below 18 years old.

In summary, the average RFNL thickness and the RFNL thickness in the superior, inferior and nasal quadrants as well as the average macular thickness and macular volume decrease as the AL and the level of myopia increase, even considering a correction of the magnification effect. Furthermore, the RFNL in the temporal quadrant is thicker in longer eyes and the central macular thickness is thicker in longer and more myopic eyes. No correlations between $\mathrm{AL} / \mathrm{SE}$ and $\mathrm{ONH}$ parameters are present after correction for magnification effect. Clinicians should be cautious when eyes with shorter or longer AL are measured and should take into account the effect of ocular magnification in order to avoid errors in the interpretation of the data from Cirrus ${ }^{\mathrm{TM}} \mathrm{HD}-\mathrm{OCT}$, regardless of age.

\section{References}

1. C. K. M. Chan, "The use of optical coherence tomography in neuro-ophthalmology," Hong Kong J. Ophthal. 15, 12-19 (2011).

2. S. C. Huynh, X. Y. Wang, E. Rochtchina, J. G. Crowston, P. Mitchell, "Distribution of optic disc parameters measured by OCT: Findings from a population-based study of 6-year-old Australian children," Invest. Ophthalmol. Vis. Sci. 47, 32763285 (2006).

3. X. Y. Wang, S. C. Huynh, G. Burlutsky, F. Stapleton, P. Mitchell, "Reproducibility of and effect of magnification on optical coherence tomography measurements in children," Am. J. Ophthalmol. 143, 484-488 (2007).
4. G. Savini, P. Barboni, V. Parisi, M. Carbonelli, "The influence of axial length on retinal nerve fibre layer thickness and optic-disc size measurements by spectral-domain OCT," Br. J. Ophthalmol. 96, 57-61 (2012).

5. V. Oner, V. Aykut, M. Tas, M. F. Alakus, Y. Iscan, "Effect of refractive status on peripapillary retinal nerve fibre layer thickness: A study by RTVue spectral domain optical coherence tomography," $\mathrm{Br}$. J. Ophthalmol. 97, 75-79 (2013).

6. M. Taş, V. Oner, F. M. Türkcü, M. F. Alakuş, A. Simşek, Y. Işcan, A. T. Yazici, "Peripapillary retinal nerve fiber layer thickness in hyperopic children," Optom. Vis. Sci. 89, 1009-1013 (2012).

7. V. Aykut, V. Öner, M. Taş, Y. Işcan, A. Ağaçhan, "Influence of axial length on peripapillary retinal nerve fiber layer thickness in children: A study by RTVue spectral-domain optical coherence tomography," Curr. Eye Res. 38, 1241-1247 (2013).

8. H. T. Lim, B. Y. Chun, "Comparison of OCT measurements between high myopic and low myopic children," Optom. Vis. Sci. 90, 1473-1478 (2013).

9. S. H. Kang, S. W. Hong, S. K. Im, S. H. Lee, M. D. Ahn, "Effect of myopia on the thickness of the retinal nerve fiber layer measured by Cirrus HD optical coherence tomography," Invest. Ophthalmol. Vis. Sci. 51, 4075-4083 (2010).

10. I. Ctori, S. Gruppetta, B. Huntjens, "The effects of ocular magnification on Spectralis spectral domain optical coherence tomography scan length," Graefe's Arch. Clin. Exp. Ophthalmol. 253, 733-738 (2015).

11. I. Bueno-Gimeno, "Cambios anatómicos en el proceso de emetropización: Influencia de las propiedades biomecánicas corneales y los parámetros anatómicos oculares," Ph.D. Thesis, Universidad Católica de Valencia San Vicente Mártir, Spain (2013), Available at: www.educacion.gob.es/teseo/ imprimirFicheroTesis.do?fichero $=45933$.

12. I. Bueno-Gimeno, E. España-Gregori, A. GenéSampedro, A. Lanzagorta-Aresti, D. P. Pinero-Llorens, "Relationship among corneal biomechanics, refractive error, and axial length," Optom. Vis. Sci. 91, 507-513 (2014).

13. K. Zadnik, D. O. Mutti, Incidence and distribution of refractive anomalies, Borish's Clinical Refraction, W. J. Benjamin, Ed., 2nd Edition, W. B. Saunders Co., Philadelphia, PA, pp. 35-55 (2006).

14. E. Arranz-Marquez, M. A. Teus, "Relation between axial length of the eye and hypotensive effect of latanoprost in primary open angle glaucoma," $B r . J$. Ophthalmol. 88, 635-637 (2004).

15. L. Liu, J. Zou, H. Huang, J. G. Yang, S. R. Chen, "The influence of corneal astigmatism on retinal nerve fiber layer thickness and optic nerve head 
parameter measurements by spectral-domain optical coherence tomography," Diagn. Pathol. 5, 55 (2012).

16. Y. H. Hwang, S. M. Lee, Y. Y. Kim, J. Y. Lee, C. Yoo, "Astigmatism and optical coherence tomography measurements," Graefes Arch. Clin. Exp. Ophthalmol. 250, 247-254 (2012).

17. J. Santodomingo-Rubido, E. A. Mallen, B. Gilmartin, J. S. Wolffsohn, "A new non-contact optical device for ocular biometry," Br. J. Ophthalmol. 86, 458-462 (2002).

18. I. Altemir, V. Pueyo, N. Elía, V. Polo, J. M. Larrosa, D. Oros, "Reproducibility of optical coherence tomography measurements in children," Am. J. Ophthalmol. 155, 171-176 (2013).

19. B. M. Burkholder, B. Osborne, M. J. Loguidice, E. Bisker, T. C. Frohman, A. Conger, J. N. Ratchford, C. Warner, C. E. Markowitz, D. A. Jacobs, S. L. Galetta, G. R. Cutter, M. G. Maguire, P. A. Calabresi, L. J. Balcer, E. M. Frohman, "Macular volume determined by optical coherence tomography as a measure of neuronal loss in multiple sclerosis," Arch. Neurol. 66, 1366-1372 (2009).

20. G. Wang, K. L. Qiu, X. H. Lu, L. X. Sun, X. J. Liao, H. L. Chen, M. Z. Zhang, "The effect of myopia on retinal nerve fiber layer measurement: A comparative study of spectral-domain optical coherence tomography and scanning laser polarimetry," $B r$. J. Ophthalmol. 95, 255-260 (2011).

21. G. Vizzeri, R. N. Weinreb, A. O. Gonzalez-Garcia, C. Bowd, F. A. Medeiros, P. A. Sample, L. M. Zangwill, "Agreement between spectral-domain and time-domain OCT for measuring RNFL thickness," Br. J. Ophthalmol. 93, 775-781 (2009).

22. H. Littmann, "Determination of the real size of an object on the fundus of the living eye," Klin. Monatsbl. Augenheilkd. 180, 286-289 (1982).

23. A. G. Bennett, A. R. Rudnicka, D. F. Edgar, "Improvements on Littmann's method of determining the size of retinal features by fundus photography," Graefes Arch. Clin. Exp. Ophthalmol. 232, 361-367 (1994).

24. A. R. Rudnicka, R. O. Burk, D. F. Edgar, F. W. Fitzke, "Magnification characteristics of fundus imaging systems," Ophthalmology 105, 2186-2192 (1998).

25. N. Elía, V. Pueyo, I. Altemir, D. Oros, L. M. Pablo, "Normal reference ranges of optical coherence tomography parameters in childhood," Br. J. Ophthalmol. 96, 665-670 (2012).

26. S. A. Vernon, A. P. Rotchford, A. Negi, S. Ryatt, C. Tattersal, "Peripapillary retinal nerve fiber layer thickness in highly myopic Caucasians as measured by Stratus optical coherence tomography," Br. J. Ophthalmol. 92, 1076-1080 (2008).

27. J. Barrio-Barrio, S. Noval, M. Galdós, M. RuizCanela, E. Bonet, M. Capote, M. Lopez, "Multicenter Spanish study of spectral-domain optical coherence tomography in normal children," Acta Ophthalmol. 91, e56-e63 (2013).

28. P. C. Wu, Y. J. Chen, C. H. Chen, Y. H. Chen, S. J. Shin, H. J. Yang, H. K. Kuo, "Assessment of macular retinal thickness and volume in normal eyes and highly myopic eyes with third-generation optical coherence tomography," Eye 22, 551-555 (2008).

29. S. C. Huynh, X. Y. Wang, E. Rochtchina, P. Mitchell, "Peripapillary retinal nerve fiber layer thickness in a population of 6-year-old children: Findings by optical coherence tomography," Ophthalmology 113, 1583-1592 (2006).

30. L. A. Ostrin, J. Yuzuriha, C. F. Wildsoet, "Refractive error and ocular parameters: Comparison of two SD-OCT systems," Optom. Vis. Sci. 92, 437-446 (2015).

31. P. J. Foster, Y. Jiang, "Epidemiology of myopia," Eye 28, 202-208 (2014).

32. D. J. Salchow, Y. S. Oleynikov, M. F. Chiang, S. E. Kennedy-Salchow, K. Langton, J. C. Tsai, L. A. AlAswad, "Retinal nerve fiber layer thickness in normal children measured with optical coherence tomography," Ophthalmology 113, 786-791 (2006).

33. M. A. El-Dairi, S. G. Asrani, L. B. Enyedi, S. F. Freedman, "Optical coherence tomography in the eyes of normal children," Arch. Ophthalmol. 127, 50-58 (2009).

34. S. C. Huynh, X. Y. Wang, G. Burlutsky, E. Rochtchina, F. Stapleton, P. Mitchell, "Retinal and optic disc findings in adolescence: A populationbased OCT study," Invest. Ophthalmol. Vis. Sci. 49, 4328-4335 (2008).

35. I. Bueno-Gimeno, A. Gene-Sampedro, D. P. PiñeroLlorens, A. Lanzagorta-Aresti, E. Espanña-Gregori, "Corneal biomechanics, retinal nerve fiber layer, and optic disc in children," Optom. Vis. Sci. 91, 1474-1482 (2014). 